\title{
Directeurs généraux et directeurs généraux adjoints des \\ Commissions scolaires (CS) du Québec
}

Un corps professionnel?

\section{Québec School Board (SB) Director Generals and Assistant}

General Directors

\section{A Professional Body?}

\section{Directores generales y directores generales adjuntos de las Comisiones escolares (CS) de Quebec ¿Un cuerpo profesional?}

\author{
Jean-J. Moisset et Jean Plante
}

Volume 32, numéro 2, automne 2004

Administrateur, administratrice scolaire et identité professionnelle

URI : https://id.erudit.org/iderudit/1079070ar

DOI : https://doi.org/10.7202/1079070ar

Aller au sommaire du numéro

\section{Éditeur(s)}

Association canadienne d'éducation de langue française

ISSN

0849-1089 (imprimé)

1916-8659 (numérique)

Découvrir la revue

Citer cet article

Moisset, J.-J. \& Plante, J. (2004). Directeurs généraux et directeurs généraux adjoints des Commissions scolaires (CS) du Québec : un corps professionnel? Éducation et francophonie, 32(2), 10-35. https://doi.org/10.7202/1079070ar

\section{Résumé de l'article}

Profession, professionnalisation et identité professionnelle, autant de concepts qui ont retenu depuis longtemps l'attention des chercheurs et ont fait irruption plus récemment dans le secteur de l'éducation. Au Québec, c'est surtout sur la profession enseignante qu'on s'est penché jusqu'ici. Mais depuis quelque temps, on a commencé à s'intéresser à la gestion et aux gestionnaires de l'éducation. Le présent article examine la problématique de l'identité professionnelle des directeurs généraux des commissions scolaires et de leurs adjoints, dont le rôle est reconnu comme stratégiquement important parmi les gestionnaires scolaires. Il expose dans un premier temps les principaux aspects conceptuels et les composantes d'un modèle d'analyse systématique de l'identité professionnelle; il présente dans un deuxième temps les données factuelles colligées par les auteurs à partir de sources documentaires pertinentes relativement à ce que sont les DG et DGA, à ce qu'ils font et à la manière dont ils le font et enfin, à la lumière des éléments caractéristiques du modèle, il tente d'apporter des éléments de réponse à la question posée plus haut.
Tous droits réservés (C) Association canadienne d'éducation de langue française, 2004
Cedocument est protégé par la loi sur le droit d'auteur. L'utilisation des services d’Érudit (y compris la reproduction) est assujettie à sa politique d'utilisation que vous pouvez consulter en ligne. 


\section{Directeurs généraux ${ }^{1}$ et directeurs généraux adjoints des Commissions scolaires (CS) du Québec : Un corps professionnel?}

\section{Jean-J. Moisset}

Faculté des sciences de l'éducation, Université Laval, Québec, (Québec), Canada.

\section{Jean Plante}

Faculté des sciences de l'éducation, Université Laval, Québec, (Québec), Canada.

\section{RÉSUMÉ}

Profession, professionnalisation et identité professionnelle, autant de concepts qui ont retenu depuis longtemps l'attention des chercheurs et ont fait irruption plus récemment dans le secteur de l'éducation. Au Québec, c'est surtout sur la profession enseignante qu'on s'est penché jusqu'ici. Mais depuis quelque temps, on a commencé à s'intéresser à la gestion et aux gestionnaires de l'éducation. Le présent article examine la problématique de l'identité professionnelle des directeurs généraux des commissions scolaires et de leurs adjoints, dont le rôle est reconnu comme stratégiquement important parmi les gestionnaires scolaires.

1. Le masculin est utilisé dans le seul but d'allègement du texte et sans préjudice aucun pour les personnes de sexe féminin. 
Il expose dans un premier temps les principaux aspects conceptuels et les composantes d'un modèle d'analyse systématique de l'identité professionnelle; il présente dans un deuxième temps les données factuelles colligées par les auteurs à partir de sources documentaires pertinentes relativement à ce que sont les DG et DGA, à ce qu'ils font et à la manière dont ils le font et enfin, à la lumière des éléments caractéristiques du modèle, il tente d'apporter des éléments de réponse à la question posée plus haut.

\section{ABSTRACT}

\section{Québec School Board (SB) Director Generals and Assistant General Directors: A Professional Body?}

Jean-J. Moisset, Faculty of Education Sciences, Laval University, Québec City, (Québec), Canada. Jean Plante, Faculty of Education Sciences, Laval University, Québec City, (Québec), Canada.

Profession, professionalizsation and professional identity are concepts that have long held the attention of researchers, and more recently erupted in the education sector. Until now in the province of Québec, it was the teaching profession that was most often scrutinized. But researchers have started to take an interest in education managers and management. This article takes a look at the issue of the professional identity of school board general directors and their assistants, whose role is recognized as being strategically important to school administrators.

The article first describes the main conceptual aspects and parts of a systems analysis model of professional identity; it then presents the factual data the authors collated from documentary sources about what director generals and assistant director generals are, what they do, and how they do it, and finally, in light of the model's characteristic elements, they attempt to introduce elements of an answer to the question asked above.

\section{RESUMEN}

\section{Directores generales y directores generales adjuntos de las Comisiones escolares (CS) de Quebec : ¿Un cuerpo profesional?}

Jean-J. Moisset, Facultad de ciencias de la educación, Universidad Laval, Quebec, (Quebec), Canadá.

Jean Plante, Facultad de ciencias de la educación, Universidad Laval, Quebec, (Quebec), Canadá.

Profesión, profesionalización e identidad profesional constituyen algunos de los conceptos que han retenido la atención de los investigadores y que reciente- 
mente han hecho su aparición en el sector de la educación. En Quebec, es la profesión de maestro la que ha atraído sobre todo la atención, aunque desde hace algún tiempo, la gestión y los gestores de la educación han despertado el interés. El presente artículo examina la problemática de la identidad profesional de los directores generales de las comisiones escolares y de sus adjuntos, cuyo rol se reconoce como estratégicamente importante entre los administradores escolares.

Se presentan en un primer momento los principales aspectos conceptuales y los elementos constitutivos de un modelo de análisis sistemático de la identidad profesional; en un segundo momento se presentan los datos factuales recogidos por los autores en fuentes documentales pertinentes relacionadas con los DG y los DGA, lo que hacen y la manera de hacerlo y finalmente, a la luz de los elementos característicos del modelo, se aportan los elementos de una respuesta a la pregunta que completa el título del artículo.

\section{Introduction}

Ce n'est pas d'hier que la notion de profession et les questions liées à la professionnalisation et à l'identité professionnelle ont retenu l'attention des chercheurs et des théoriciens. Cela est particulièrement vrai pour les sociologues qui ne pouvaient s'empêcher de mettre en perspective ces problématiques spécifiques (identité professionnelle) par rapport à la problématique générale de la socialisation et de l'identité sociale, leur objet privilégié d'études, dirait-on, de toujours. À cet égard, sans remonter au déluge et pour ne citer que ceux-ci, on peut mentionner les chefs de file des grands courants théoriques qui ont traversé ce champ : Margaret Mead (1934) avec l'interactionnisme symbolique, Talcott Parsons (1969) avec le structuro-fonctionnalisme et plus près de nous Pierre Bourdieu (1987) avec l'approche critique.

Sans doute, à cause entre autres de l'important rôle dévolu aux systèmes d'éducation formelle dans la socialisation des individus et leur accession à une profession, les chercheurs se sont très tôt intéressés aux activités qui s'y déroulent. Emile Durkheim lui-même, au début du siècle dernier, s'est penché sur les rapports entre l'éducation et la sociologie. Et même si on ne peut parler de filiation directe, on peut dire que dans cette perspective s'est constituée une abondante littérature portant essentiellement sinon exclusivement sur «le métier d'enseignant», «la professionnalisation enseignante ", «l'enseignement et le professionnalisme». Elle illustre, sans contredit, l'importance accordée à «l'éducation comme profession" (Lieberman, 1956) et à «l'éducation pour les professions" (Nelson, 1962). Cette problématique générale du statut professionnel des principaux acteurs oeuvrant dans le secteur de l'éducation s'est donc développée depuis plusieurs décennies et notamment dans les pays anglo-saxons. Mais, comme on va le voir, elle a fait son entrée au Québec et avec un intérêt sans cesse croissant, voilà déjà une dizaine d'années. 


\section{Problème et objectifs de l'étude}

Le Québec n’a certes pas été de reste par rapport à ce dynamisme de la recherche sur la profession enseignante, le signal de départ, peut-on dire, ayant été donné par le Rapport annuel 1990-1991 du Conseil supérieur de l'éducation (1991). Certes, dans la foulée de ce rapport, peu de temps après sa publication, la Revue des sciences de l'éducation, sous la direction de Lessard, Perron et Bélanger (1993), consacre un de ses numéros au thème de la "professionnalisation de l'enseignement et de la formation des enseignants ». Et depuis, la problématique de la formation des maîtres et de l'identité professionnelle des enseignants n'a cessé d'alimenter la recherche et les publications (Féger (dir.), 1997; Desaulmiers et al. (dir.), 1997; Tardif et Ziarko (dir.), 1997; Gohier et al. (dir.), 2000; Gohier et al., 2001).

Si de manière générale, et au Québec en particulier, les recherches sur la professionnalisation en milieu scolaire se sont surtout penchées sur les enseignants, il n'en reste pas moins, selon le Conseil supérieur de l'éducation (1991), que la reconnaissance du caractère professionnel de l'acte d'enseigner a été en butte à plusieurs obstacles, dont certains liés aux modes de gestion des organisations scolaires. De manière même indirecte et en l'occurrence négative, un autre groupe occupationnel, celui des administrateurs scolaires, était ainsi introduit dans les débats. Deux années plus tard, c'est directement que ce même groupe, large, des administrateurs scolaires sera interpellé par le Conseil supérieur de l'éducation (1993), soulignant «la nécessité d'un autre modèle de gestion de l'éducation".

Ces observations sont dans un certain sens une forme de reconnaissance de l'importance de ce que font les administrateurs scolaires et de la manière dont ils le font, ce qui n'est pas sans lien avec ce qu'ils sont. Il est certes de plus en plus reconnu que l'organisation et la gestion scolaire, à quelque niveau où l'on se situe dans le système éducatif, mais particulièrement au palier intermédiaire et à la base, sont lourdement responsables des conditions et du climat dans lesquels se déroule l'acte d'enseigner. Elles constituent ainsi des facteur significatifs de la réalisation des missions de l'école, voire de la réussite scolaire des élèves. Cela a été souligné par le Conseil supérieur de l'éducation (1993, p. 11) souhaitant que «la gestion de l'éducation [soit] passée au crible de l'analyse, tellement on lie la qualité de la formation dispensée et l'efficacité de la mise en œuvre même des services". Et dans cette perspective d'un autre modèle de gestion, où «le gouvernement des personnes aurait sensiblement plus de place que l'administration des choses » (idem, p. 10), la révision des rôles des gestionnaires, entre autres, s'impose comme une tâche essentielle, soulignait-on.

Dans le contexte actuel et un peu plus de dix ans après la parution de ce rapport du Conseil, il nous a paru pertinent d'examiner la problématique de l'administration scolaire à partir de ceux qui la pratiquent. Nous nous intéressons de manière spécifique aux directeurs généraux et directeurs généraux adjoints des commissions scolaires, dont nous tâcherons d'examiner les rôles et les fonctions ainsi que leurs modes et conditions d'exercice. 
Environ trois décennies après la Réforme Parent, le système scolaire du Québec est de nouveau l'objet de changements importants. Au plan administratif, avec l'actuelle réforme, on assiste à une redistribution des pouvoirs entre le palier intermédiaire et la base, allant dans le sens d'une plus grande autonomie de l'établissement scolaire. Auparavant, les commissions scolaires et leurs diverses instances avaient connu d'importantes transformations, ce qui n'a pas été sans influencer les rôles et fonctions des directeurs généraux et de leurs adjoints. En outre, au cours des dernières décennies, les directeurs généraux, bras droits des Conseils des Commissaires se dont dotés d'une structure associative, l'ADIGECS (Association des directeurs généraux des commissions scolaires) dont l'une des importantes missions est "d'assurer la qualité de l'exercice de la profession en soutenant le développement et le perfectionnement de ses membres et en contribuant à la détermination de normes professionnelles d'exercice de la profession ». (ADIGECS, 2002)

De fait, le mois de mai de l'année 2002 a ramené le trentième anniversaire de la création de l'ADIGECS, raison conjoncturelle supplémentaire qui nous a amenés à porter notre attention sur le groupe des directeurs généraux et des directeurs généraux adjoints. Les premières réflexions systématiques, dont nous a donné l'opportunité le colloque dans le cadre du $70^{\mathrm{e}}$ Congrès de l'ACFAS, portent sur la problématique de l'identité professionnelle de ces administrateurs scolaires.

L'objet du texte qui suit, découlant de notre communication, sera dans un premier temps d'exposer les aspects conceptuels généraux doublés d'un modèle d'analyse sur l'identité professionnelle que nous avons élaboré; dans un deuxième temps, seront présentées les principales données factuelles que nous avons pu colliger à partir de sources documentaires pertinents concernant les DG et les DGA et, dans un troisième et dernier temps, ces données seront analysées à la lumière des concepts et du modèle relatifs à l'identité professionnelle, ce qui nous permettra d'examiner si ces derniers constituent un groupe professionnel ou en voie de professionnalisation. Pour des raisons indépendantes de notre volonté, l'enquête sur les données perceptuelles auprès de DG et des DGA n'a pas pu être effectuée, ce qui, nous en convenons, relativise et rend encore davantage provisoire notre conclusion.

\section{La profession d'administrateur scolaire : éléments d'un modèle d'analyse de l'identité professionnelle}

\section{Complexité du phénomène d'identité}

La notion d'identité est inséparable de la problématique de la socialisation dont nous avons souligné, dès l'abord, l'importance des liens avec l'éducation. Elle est également incontournable par la réalité qu'elle recouvre et que l'on considère comme une condition sine qua non de la stabilité, même relative, indispensable à l'existence des individus, des groupes et des sociétés. Au commencement, peut-on dire à cet égard, il y a le "moi», si magnifiquement illustré par Descartes dans son célèbre " cogito, ergo sum", "Je pense, donc je suis». Mais qui suis-je? Interrogation qui n'a cessé de hanter l'esprit des penseurs, à laquelle certes ne peuvent être données des 
réponses une fois pour toutes car, souligne Erikson (cité par Dubar, 1991, p. 112), «l'identité n'est jamais installée, jamais achevée, puisque l'environnement du moi est mouvant».

Par ailleurs, à supposer que chacun, à chaque moment, soit en mesure de répondre à la question qui il est, il ne peut s'empêcher de se demander si cette «identité pour soi », pour reprendre les catégories de Dubar (idem, p. 112), correspond à "l'identité pour autrui ", c'est-à-dire à la représentation qu'ont de lui ceux avec lesquels il est en relation. Il y a donc une négociation permanente entre le «soi » et «autrui » et dont l'enjeu est l'identité sociale, la reconnaissance de soi par autrui.

Mais cette identité sociale, elle-même n'est pas univoque et exclusive d'une seule dimension de l'existence, si importante soit-elle. On naît d'un père et d'une mère, appartenant à une famille; avec sa famille, père, mère et peut-être frères et sœurs, on fait partie d'un groupe plus large : communauté locale (clan, tribu), régionale, nationale; on a son groupe d'amis; on devient élève d'une école, étudiant d'un collège ou d'une université; on acquiert un métier, une profession, constituant avec ceux qui partagent la même activité en termes d'occupation, un corps de métier, un groupe professionnel, etc. L'identité comporte donc de multiples instances.

Tout cela traduit la complexité du phénomène d'identité, à la fois facteur de permanence et de stabilité et variable dans le temps; oscillant entre ce qu'on est pour soi-même et ce qu'on est pour les autres; déterminant du caractère unique de chaque individu et cependant ouvert à la pluralité. Cette complexité du phénomène se reflète bien à travers cette définition qu'en propose Dubar (1991, p. 113), à savoir que "l'identité n'est rien d'autre que le résultat à la fois stable et provisoire, individuel et collectif, subjectif et objectif, biographique et structurel, des divers processus de socialisation qui, conjointement, construisent les individus et définissent les institutions». Cette complexité de l'identité sociale n'a pas moins permis aux multiples recherches engagées depuis longtemps d'aboutir à un certain nombre d'idées convergentes sinon de certitudes qui ont fait leur entrée dans l'étude des professions, de la professionnalisation et de l'identité professionnelle. Ce sera l'objet de la section qui suit que de les identifier et de les mettre en relief en les définissant, avant d'en dégager le potentiel, comme outil d'analyse applicable au cas des directeurs généraux et des directeurs généraux adjoints des commissions scolaires.

\section{De l'identité sociale à l'identité professionnelle : quelques concepts clefs}

Nous référant à ce qui précède, nous pouvons dire, dans un premier temps tout au moins, que l'identité professionnelle est une instance ou une dimension particulière de l'identité sociale. Comme telle, elle fait intervenir les mêmes éléments de complexité mis en relief auparavant, entre autres l'ambivalence entre le permanent et le variable, et les jeux d'interaction entre la représentation que l'on a de soi et l'image que l'on projette chez les autres, les interrelations entre la perspective individuelle et la perspective collective. L'identité professionnelle se distingue néanmoins nettement de l'identité sociale, dans la mesure où elle réfère, pour un individu ou un groupe, aux rôles, aux fonctions et aux tâches assumés dans un champ donné d'ac- 
tivités et aux structures et mécanismes formels et informels (mais surtout formels) balisant ce champ d'activités. Elle est donc spécifique à une seule sphère de l'existence humaine et, comme telle, n'est qu'une composante de l'identité sociale.

À la base de l'identité professionnelle, il y a des réalités caractéristiques d'un travail donné et du milieu ou de la situation de ce travail que traduisent quelques concepts majeurs, devenus incontournables, tant ils ont été étudiés et repris successivement par tous les chercheurs qui se sont penchés sur cette problématique. Pour notre part, et sans prétendre en cela à une quelconque originalité, nous mettrons en relief dans ce qui suit, les trois plus importants à nos yeux, soit les concepts de " profession», de «professionnalisation» et de "professionnalisme» avant de revenir sur le concept intégrateur d'identité professionnelle. L'on ne sera pas étonné cependant que cette exposition des concepts, procédant de choix qui nous sont propres, ne coïncide pas avec tout ce que la littérature offre sur l'éventail plutôt large et diversifié des questions qui s'y rattachent.

\section{La notion de profession}

Selon les auteurs se rattachant à la sociologie fonctionnaliste ou plus spécifiquement à la sociologie classique du travail, la notion de profession se confond avec les attributs spécifiques qui la caractérisent. Au premier chef, une activité professionnelle, une profession est supposée répondre à des problèmes ou des besoins particuliers ressentis par une collectivité. Cette perspective a été développée notamment par plusieurs chercheurs Nord-Américains dans la période qui a suivi la seconde guerre mondiale, à la suite des pionniers Flexner (1915) et Carr-Saunders et Wilson (1933). Qu'il s'agisse de Goode (1957; 1960), Gross (1958), de Blau et Scott (1962), de Becker (1962), pour ne citer que ceux-là, leurs idées convergent vers une conception des professions perçues comme des produits ayant en commun un certain nombre de traits que l'on peut identifier. De fait, leurs travaux offrent, pour la plupart, des listes aux nombres variés de caractéristiques, qui se recoupent généralement pour l'essentiel, permettant de définir une profession. Ainsi, Lieberman (1956) ${ }^{2}$ "propose huit caractéristiques à prendre en compte pour définir la profession". Celle-ci doit accomplir un service essentiel et unique pour la société, au moment où le service est fourni; elle doit mettre l'accent sur des techniques à caractère intellectuel. Ce type d'occupation suppose une longue période de formation spécialisée, la possession d'une grande autonomie de la part des individus qui l'exercent, l'acceptation d'un grand degré de responsabilité et la présence d'un code d'éthique et de certaines normes et de certains règlements relatifs à la certification et au maintien des standards de pratiques".

À la différence de cette nomenclature de Lieberman, Goode (1957) propose une perspective analytique qui perçoit les professions comme des communautés à l'intérieur d'une communauté plus large, répondant à des critères tels que l'identité commune, les définitions de rôles, l'engagement et les valeurs. De surcroît, il en vien-

2. Cité par Marta Anadon (2000). L'enseignement en voie de professionnalisation. Dans Christiane Gohier et al. (dir.), L'enseignant un professionnel, Ste-Foy (Québec), Presses de I'Université du Québec, p. 4. 
dra plus tard (1960, p. 903) à différencier ces critères selon leur degré d'importance, «les deux principales caractéristiques des professions, selon lui, celles autour desquelles s'articulent toutes les autres, étant l'acquisition d'une formation spécialisée et prolongée dans une branche du savoir abstrait et l'intérêt manifesté envers la collectivité et le service». Cela dit, il n’aligne pas moins de dix traits qu'une occupation acquiert au fur et à mesure qu'elle se professionnalise, à savoir que :

1. la profession détermine ses propres standards de formation;

2. l'étudiant aspirant professionnel passe à travers une expérience de socialisation adulte de bien plus grande portée (envergure) que l'apprenant dans d'autres champs occupationnels;

3. la pratique professionnelle est généralement reconnue légalement par une forme ou une autre de licence (permis);

4. l'attribution des permis et l'admission dans l'ordre professionnel sont régies (gérées) par les membres de la profession;

5. la plupart des législations touchant une profession sont façonnées par la profession même;

6. plus une profession gagne en termes d'échelle de revenu, de pouvoir et de prestige, plus elle est exigeante concernant le calibre des candidats-aspirants;

7. le praticien d'une profession n'est généralement pas soumis au contrôle et à l'évaluation de profanes (entendons autres que ceux de son ordre professionnel);

8. les normes de la pratique imposées par une profession sont plus contraignantes que les contrôles légaux;

Dans une profession, le contrôle du travail par les membres renvoie surtout au fait que le travail n'est pas supervisé par des profanes extérieurs à leur groupe occupationnel.

9. le sentiment d'identification et d'appartenance des membres à leur ordre professionnel est généralement beaucoup plus fort que chez les membres d'autres groupes occupationnels;

10. une profession a plus de chance d'être une occupation finale (terminale), que les membres ne quittent généralement pas et dont une proportion très élevée affirme qu'ils la choisiraient encore s'ils avaient à le faire.

Définitivement, l'approche de Goode, à travers cette liste de dix caractéristiques, a l'avantage, d'une part, d'illustrer, de manière plus explicite, la vision fonctionnaliste des professions, par définition au service de la collectivité et dans un domaine particulier. C'est le quasi monopole des savoirs et savoir-faire détenus par les membres qui justifie la très large autonomie et la liberté dont ils jouissent dans l'exercice de leur profession. Encore faut-il que les normes imposées par la profession soient contraignantes et respectées, l'intérêt du public transcendant les intérêts particuliers de l'ordre professionnel et de ses membres. À cet égard, nous rejoignons Trottier (1999, pp. 7-20) quand il souligne que «dans une profession, le contrôle du travail par les membres renvoie surtout au fait que le travail n'est pas supervisé par des profanes extérieurs à leur groupe occupationnel. Parce qu'ils détiennent une compétence exclusive dans une branche du savoir et qu'ils intériorisent les normes qui doivent présider l'exécution des tâches, une large responsabilité leur est déléguée, et ils bénéficient d'une certaine autonomie». 
Il n'en est pas moins vrai que, comme une arme à double tranchant, ce monopole sur un domaine particulier peut comporter des risques sérieux pour la collectivité, de sorte que certains mécanismes formels voire un encadrement juridique soient nécessaires parallèlement « au contrôle social informel [même lorsqu'il] apparaît d'abord comme une garantie des pratiques professionnelles ", pour reprendre les mots de Trottier. La quintessence de cette vision se résumerait au fait qu'une profession est une occupation formelle reconnue comme un ordre professionnel par une instance légale prévue à cet effet.

Ainsi nous permettra-t-on de citer les cinq (5) critères que le Code des professions du Québec (article 25) prévoit pour recommander la création d'un ordre professionnel.

Ils portent respectivement sur:

1. les connaissances requises des personnes pour exercer les activités;

2. l'autonomie dont jouissent les membres de cet ordre dans l'exercice de leurs activités professionnelles;

3. le caractère personnel des rapports entre ces personnes et les gens recourant à leurs services, en raison de la confiance particulière que ces derniers sont appelés à leur témoigner;

4. les préjudices ou dommages que pourraient subir les gens recourant aux services de ces personnes par suite du fait que leur compétence ou leur intégrité ne seraient pas contrôlées par l'ordre;

5. le caractère confidentiel des renseignements que ces personnes sont appelées à connaître dans l'exercice de leur profession.

Il nous semble clair, tout en reconnaissant l'importance de l'ensemble de ces critères centrés sur la protection du public potentiellement bénéficiaire de ses services, que la vision fonctionnaliste de la profession ne rend pas compte explicitement de la dynamique fort complexe des interrelations entre les individus, les groupes et la société dans le processus de construction de l'identité professionnelle. C'est cette dimension que semble, à première vue, prendre en charge l'approche interactionniste symbolique que nous associons au concept de professionnalisation qui est examiné dans ce qui suit.

\section{Le concept de professionnalisation}

L'approche interactionniste symbolique reconnaît les professions comme des réalités dont on peut mettre en relief la substance et les attributs propres. Mais ces réalités sont relatives ou plus ou moins objectives, parce qu'objectivées à un moment donné et donc susceptibles de changement dans un processus interactif dynamique entre les représentations que les individus ont de leur occupation et de leurs pratiques et les représentations que s'en font les autres, autres individus, groupes et collectivité. Dans cette optique, il est aussi important de connaître un produit que sa genèse, d'autant plus que ce produit, en l'occurrence la profession, ne peut être qu'un état transitoire, quelle que soit la durée de cette transition, d'un processus jamais 
achevé. L’on rejoint ainsi la notion de "profession, reconnue comme un ensemble de pratiques sociales et définie par des rôles et des conduites diverses contribuant à faire évoluer les identités des individus» (Cohen-Scali, 2000, p. 86).

Si la professionnalisation est généralement reconnue comme un processus dont l'aboutissement est l'émergence d'une profession, l'envergure et la portée de ce processus sont saisies de manière différente selon les auteurs. D'aucuns parlent d'éléments constitutifs de la professionnalisation, comme Jenkins qui, cité par Anadon (idem, p. 7), en distingue six majeurs : structurel, contextuel, relatif à l'activité, idéologique, éducationnel et comportemental. On peut faire remarquer que ces éléments constitutifs de la professionnalisation ne sont pas bien loin des attributs spécifiques de la profession, avec lesquels ils partagent le caractère plus ou moins statique et descriptif. Et c'est également le cas pour « les caractéristiques communes à toutes les occupations » ou " les facteurs de différenciation des professions » de Hughes, égale-

La notion de professionnalisation qui se dégage de ce qui précède est celle d'une suite d'actions entreprises par un groupe d'individus liés à et par une commune occupation en vue de se doter de certaines caractéristiques. ment rapportées par Anadon (idem, p. 8). C'est enfin le dilemme auquel ont été confrontés les auteurs qui ont réduit la professionnalisation à l'établissement d'une typologie des professions ou d'une classification selon des niveaux. Nous citerons encore Marta Anadon qui rapporte (idem, p. 9) que Ritzer et Walczakk avaient identifié dans leur étude "cinq niveaux dans le processus de professionnalisation: les professions marginales, les occupations qui aspirent à la professionnalisation, les semi-professions, les nouvelles professions et les professions traditionnellement établies ".

La notion de professionnalisation qui se dégage de ce qui précède est celle d'une suite d'actions entreprises par un groupe d'individus liés à et par une commune occupation en vue de se doter de certaines caractéristiques, leur ultime visée étant leur reconnaissance comme un groupe professionnel distinct et mieux, comme un ordre professionnel au sens du Code des professions. À cet égard, l'on soulignera à la suite de ces auteurs que trois éléments majeurs marquent le processus de professionnalisation à savoir: "l'uniformisation des pratiques assorties de conditions spécifiques d'exercice de la profession; la définition de normes et de standards; l'exclusivité de services avec contrôle sur l'ensemble de la pratique et la formation en conséquence des membres». Il va de soi que la mise en œuvre de ces actions s'accompagne de toute une série d'interactions entre les individus au sein du groupe et entre le groupe et la communauté susceptibles de faire émerger un sentiment d'identification et d'appartenance au groupe de référence. En dépit de tout cela, comme nous le mettrons plus loin en relief, la professionnalisation ne recouvre pas le processus de construction de l'identité professionnelle. Mais examinons auparavant la notion de professionnalisme.

\section{Le concept de professionnalisme}

Alors que la professionnalisation est un processus relatif à une occupation donnée et au groupe de personnes qui y sont associées, le professionnalisme renvoie aux individus qui, dans l'accomplissement de leurs activités professionnelles, sont aptes à démontrer leur maîtrise des savoirs et des savoir-faire définissant leur champ professionnel ainsi que du savoir-être, notamment en termes de valeurs, de normes et 
d'attitudes, qui y est relié. C’est le sens généralement donné aux expressions courantes «faire preuve de professionnalisme», «être professionnel ou professionnaliste», l'accent étant cependant mis sur le savoir-être, ce qui n'entraîne nullement une diminution de l'importance de compétences spécifiques dans la perspective de la construction d'une identité professionnelle. L’on trouve dans ce concept ainsi défini ce par quoi, dirait Goode (1960), on peut distinguer les vrais professionnels des usurpateurs et des charlatans.

Il est ainsi difficile en traitant du professionnalisme de ne pas faire état de l'éthique au sens de valeurs fondamentales et de normes spécifiques à respecter, d'attitudes et de comportements à afficher, ce qui suppose qu'elles ont été préalablement intériorisées. Le professionnalisme peut être donc considéré autant comme un ensemble de qualités, de façons d'être que le processus au terme duquel elles ont été acquises et développées. À ce point de vue, le professionnalisme pourra être apprécié suivant des degrés distribués sur une échelle et attribuables aux personnes exerçant les fonctions reliées à cette profession.

L'ensemble des éléments précédents, à quelque école de pensée à laquelle ils se rattachent, convergent pour donner lieu à l'identité professionnelle dont nous tâcherons, dans la section suivante, de présenter une définition ainsi qu'un modèle d'analyse.

\section{Concept et modèle d'analyse de l'identité professionnelle}

Identité professionnelle : le concept

À l'instar de l'identité sociale, l'identité professionnelle se présente à travers la littérature comme une réalité éminemment complexe découlant d'un processus très long et multi-forme, faisant intervenir de nombreuses interactions entre divers acteurs, individuels et collectifs. Dans une acception première, elle est d'ailleurs saisie comme " une forme particulière d'identité sociale qui se manifeste dans et par l'implication au travail» (Cohen-Scali, 2000, p. 82). Mais la définition qu'en donne Claude Dubar (1996, p. 111), déjà citée, fait encore mieux ressortir la complexité de l'identité professionnelle, selon lui, « résultat à la fois stable et provisoire, individuel et collectif, subjectif et objectif, biographique et structurel, des divers processus de socialisation qui, conjointement, construisent les individus et définissent les institutions ». Cela dit, nous laisserons de côté la dynamique proprement dite des processus liés à la construction pour centrer notre attention sur les principales composantes de l'identité professionnelle, dont nous tâcherons d'en faire une brève synthèse.

Plusieurs définitions opérationnelles sont offertes de l'identité professionnelle. Ainsi Valérie Cohen-Scali (2000, p. 90 et ss), à la suite de plusieurs chercheurs ayant étudié l'identité professionnelle la conçoit comme «une accumulation de savoir-faire et d'habiletés, associés à une profession". Attitudes des individus à l'égard d'euxmêmes, représentations sociales communes aux membres et reflétant le savoir commun des individus sur eux-mêmes sont autant d'éléments qui, appliqués aux activités liées à un travail, deviennent constitutifs de l'identité professionnelle. Elles se traduisent alors par des pratiques de travail, des facteurs culturels, des systèmes de normes et de valeurs appuyés sur un savoir professionnel, le tout étant partagé par 
les membres du groupe. À ces éléments liés aux individus - membres et au groupe professionnel s'ajoutent ceux provenant des autres et plus globalement de la société, soit l'image que ces derniers se font de cette profession et les attentes ou voire les exigences qu'ils expriment à son égard.

On le voit, l'identité professionnelle comme résultante de ces négociations constantes entre l'individu, les membres de son groupe professionnel et la société s'articule, comme le soulignent Gohier et al. (1999) autour de trois dimensions majeures : les aspects proprement psycho-individuels, les attentes de la société et les aspects associés au groupe professionnel, qui rejoignent les trois dimensions du modèle de Cohen-Scali (2000, p. 130), sous les dénominations: dimensions personnelles, dimensions sociales et milieu de travail et de formation, dont nous nous inspirons pour élaborer notre propre modèle d'analyse de l'identité professionnelle.

Figure 1. Identité professionnelle : le modèle d'analyse

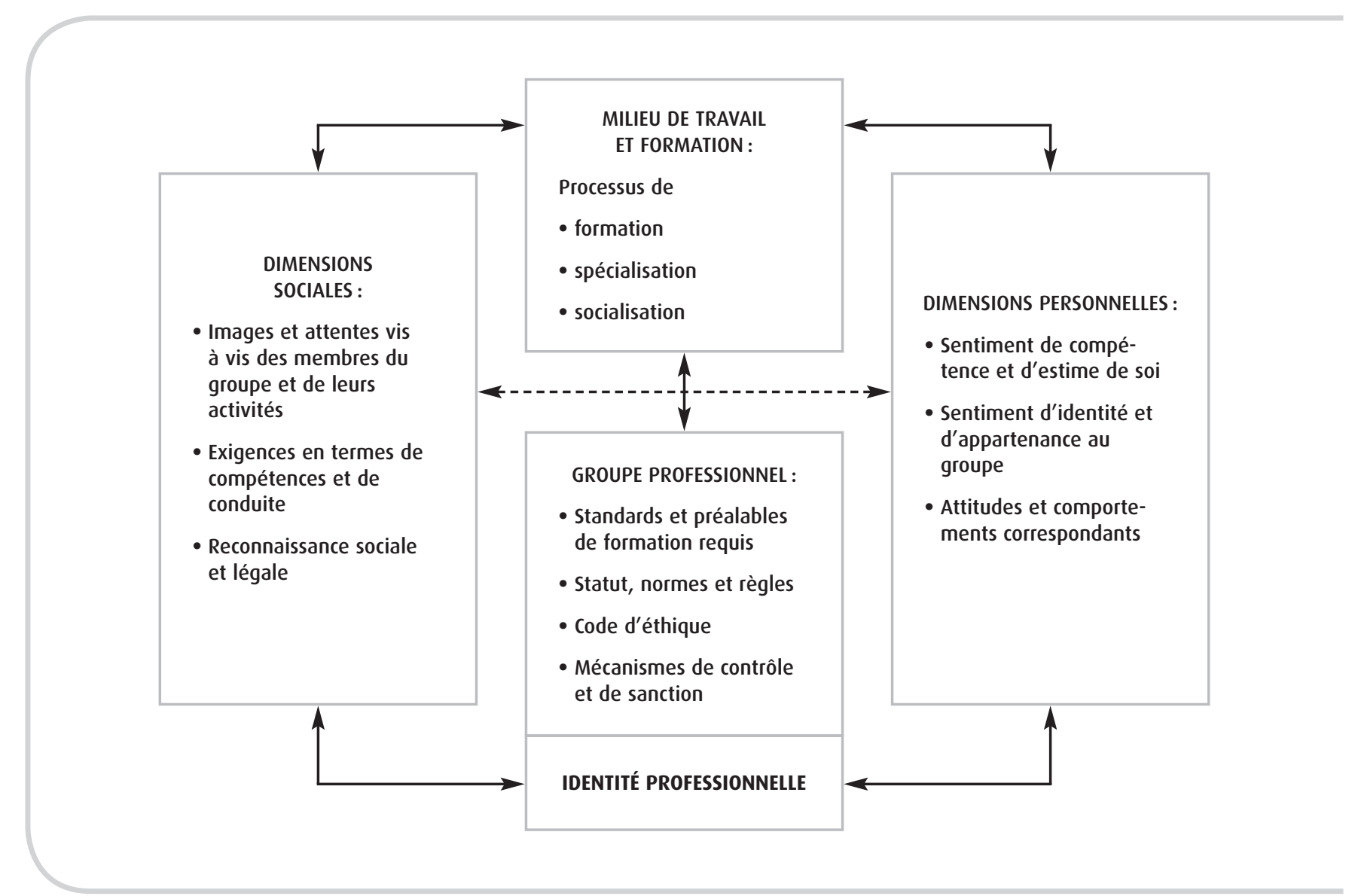

Ce schéma résume et illustre l'identité professionnelle dans ses différentes dimensions, principalement personnelles et sociales, et dans sa genèse, traduite par un ensemble d'interactions-transactions au sein du milieu de travail et du groupe professionnel. Tous les éléments de ce schéma ainsi que les lignes qui les relient, les 
doubles flèches indiquant les interactions réciproques entre eux, traduisent le caractère dynamique et complexe de la construction à laquelle ils concourent, de l'identité professionnelle, elle-même, réalité toujours en mouvement, compte tenu des évolutions de contexte et de conjoncture.

Le schéma nous aura permis d'identifier les principaux éléments à prendre en considération dans l'élaboration de l'outil de cueillette des données relativement à la fonction de directeur général et de directeur général adjoint des commissions scolaires du Québec. Il nous servira par ailleurs d'instrument d'analyse de ces données. Ces observations nous amènent à dire quelques mots de la démarche méthodologique suivie.

\section{Démarche méthodologique}

À la lumière des éléments conceptuels et du modèle d'analyse présentés plus haut, deux types d'information nous ont paru nécessaires pour pouvoir apporter des éléments de réponse relativement au statut professionnel des directeurs généraux et des directeurs généraux adjoints des commissions scolaires du Québec: des données plus factuelles d'une part et des données plus perceptuelles d'autre part.

En ce qui concerne le premier type de données, nous avons été amenés à procéder à une analyse des principaux documents pertinents, notamment des textes traitant de l'historique des commissions scolaires et de leur direction générale, des textes législatifs portant sur les fonctions-attributions des directeurs généraux des commissions scolaires et des règles encadrant leurs activités et de documents plus spécifiques ayant trait à l'Association des directeurs généraux des commissions scolaires (ADIGECS) du Québec.

Un questionnaire a été élaboré par la suite, sur la base des éléments constitutifs majeurs de notre cadre d'analyse, devant nous permettre de collecter les informations pertinentes pour compléter d'une part les données factuelles de base, relatives aux 70 directeurs généraux des commissions scolaires et de leurs adjoints et pour recueillir d'autre part, les perceptions et points de vue des DG et des DGA quant à divers aspects de leurs fonctions. Le questionnaire au total comportait 75 questions, dont quatre ouvertes, articulées autour de quatre parties et réparties comme suit:

- la première, avec 9 questions fermées portant sur les profils socio-démographique et socio-professionnel des DG et des DGA.;

- $\quad$ la deuxième, avec 23 questions, dont 22 fermées, portant sur les rôles, fonctions et responsabilités des répondants;

- la troisième, avec 25 questions, dont 24 fermées, portant sur la manière dont les répondants caractérisent et perçoivent leur fonction comme DG ou DGA.;

- la quatrième, avec 18 questions, dont 16 fermées, portant sur la manière dont les répondants se perçoivent, eux et leurs pairs, en regard de leur fonction. 


\section{Présentation et analyse des données}

\section{Les directions générales des commissions scolaires : rétrospective historique}

Afin de permettre une meilleure compréhension des données qui sont présentées ci-après, il nous apparaît important de situer historiquement le corps professionnel que constituent les directeurs généraux des commissions scolaires. On s'en doute, l'importance de ce groupe professionnel évolue en fonction des responsabilités que l'État impute à son employeur, la commission scolaire. Il convient, en conséquence, de brosser un tableau synthèse de cet organisme responsable de l'éducation préscolaire, de l'enseignement primaire et secondaire, ainsi que de l'éducation des adultes et de la formation professionnelle.

Les commissions scolaires sont mises en place au milieu du XIX ${ }^{\mathrm{e}}$ siècle. En 1948-49, on en compte 1927 et le secrétaire-trésorier est alors le haut-fonctionnaire de la commission scolaire dont les membres sont élus par les contribuables de la municipalité. Dans les milieux urbains, elles offrent aussi l'enseignement secondaire, mais dans les milieux ruraux, l'enfant doit se satisfaire de l'école primaire. Du côté anglophone, l'école secondaire donne accès à l'université alors que, chez les francophones, l'école privée, le collège classique, débouche sur l'enseignement supérieur.

Le système éducatif québécois vit sa première révolution scientifique lorsque Roland Vinette publie les résultats de sa thèse doctorale dans le Journal de l'instruction publique. Il annonce ce qui sera la pierre angulaire des programmes d'études du primaire (1948) et du secondaire (1956), ainsi que ceux de la formation des maîtres (1953). La psychologie expérimentale fait alors son entrée dans le monde de l'éducation québécois.

La révolution tranquille des années 1960 accentue la prise en charge de l'éducation obligatoire par l'État et de la responsabilisation des mécanismes politiques régionaux. À la suite du rapport de la Commission Tremblay (1956) et dans la foulée de la Commission Parent (1963-1966), le gouvernement québécois oblige les commissions scolaires à offrir l'enseignement secondaire. Cependant, force est d'admettre que les commissions scolaires ne sont ni prêtes ni équipées pour répondre à cette demande. Le gouvernement met alors en branle une gigantesque opération ${ }^{3}$ straté- $^{-}$ gique qui le conduit à l'installation de commissions régionales qui prennent charge de l'enseignement secondaire, de l'enfance inadaptée et de l'éducation des adultes. Selon Kirouac (1976), le gouvernement québécois propose alors, à ces commissions scolaires régionales, un système de gestion dans lequel deux modèles d'organisation sont présentés. De façon générale, on voit apparaître le directeur général comme premier haut-fonctionnaire de la commission régionale.

Dans la foulée de la mise en place de ces organismes régionaux, la Fédération des commissions scolaires catholiques du Québec lance, en 1968, l’Opération-regrou-

3. Connue sous le nom "Opération 55», cette politique visait l'implantation de 55 régionales catholiques sur le territoire québécois. À la fin de l'opération, en 1965, le Québec comptait 55 commissions régionales pour catholiques et 9 commissions régionales pour protestants. 
pement et incite les commissions scolaires catholiques à se fusionner. Ce mouvement est complété par l'adoption, en 1971, d'une loi ordonnant le regroupement des commissions scolaires. De 1788 qu'elles étaient, en 1960-61, on en compte 259, au premier juillet 1973.

Au cours de cette même période, il faut noter une modification majeure du mode de nomination des commissaires. De 1845 à 1972, ces représentants de la population sont élus par les contribuables, c'est-à-dire par les propriétaires qui paient la taxe scolaire, sauf pour les commissions scolaires confessionnelles de Montréal et de Québec où ils sont nommés par le gouvernement, les autorités religieuses et municipales. Depuis 1971 et 1973, ils sont élus au suffrage universel.

La participation à la gouverne de l'école et des diverses instances de la commission scolaire constitue un autre changement majeur de l'organisation scolaire québécoise. C'est ainsi que l'État québécois après avoir reconnu, dans le préambule de la loi instituant le ministère de l'Éducation et le Conseil supérieur de l'éducation, le droit des parents de choisir les institutions qui, selon leur conviction, assurent le mieux le respect des droits de leurs enfants, explicite ce droit en leur donnant une voix dans la gestion de l'école. Il met alors en place deux comités consultatifs auprès de l'école et de la commission scolaire. De 1971 à 1979, ces deux organismes consultatifs ont principalement comme mission de promouvoir la participation des parents à la gestion de l'école et de la commission scolaire. Le mouvement est suffisamment fort qu'il conduit la Commission des écoles catholiques de Montréal à mettre sur pied un comité chargé d'étudier la gestion participative de l'école secondaire.

En 1979, le gouvernement modifie la Loi sur l'instruction publique et institue le conseil d'orientation. Selon les dispositions de la loi, il est loisible à chaque école de mettre sur pied un conseil d'orientation dont le rôle consultatif porte sur des objets sur lesquels les parents, les enseignants et la direction d'école se sont entendus. Au cours des deux décennies suivantes, la gestion participative va graduellement s'installer à l'intérieur de l'école et de la commission scolaire. Enfin, lors de la dernière modification majeure de la Loi sur l'instruction publique, en 1998, le gouvernement péquiste, après avoir obtenu des modifications à la constitution canadienne, abolit la confessionnalité des commissions scolaires et met en place un conseil d'établissement auquel il accorde certains pouvoirs qui étaient jadis ceux de la commission scolaire. Dans ce même mouvement, le gouvernement regroupe les commissions scolaires: 60 francophones, 9 anglophones et 3 particulières.

Afin de permettre à l'établissement scolaire de bien s'acquitter de sa mission, l'État québécois, après moult consultations publiques et recherches de toutes sortes, installe une structure d'autorité à la fois hiérarchique et démocratique. Au sommet de la pyramide, on retrouve l'Assemblée nationale, le ministère de l'Éducation et le Conseil supérieur de l'éducation. À ce niveau, l'État s'est octroyé la responsabilité de définir les grandes orientations du système d'enseignement. Au niveau régional, là où agissent les commissions scolaires et le comité consultatif de parents, l'État a réservé le double rôle de traduction des décisions prises par les organes centraux et de surveillance de leur exécution sur leur territoire. Enfin, comme c'est en classe que se réalise concrètement la relation enseignant-apprenant, l'État a chargé l'établisse- 
ment de l'obligation d'un agir professionnel qui tient compte des besoins de l'élève et de la communauté dans laquelle s'insère l'établissement scolaire. En somme, au cours des quarante dernières années, l'État québécois s'est doté d'un système éducatif pour l'enseignement primaire et secondaire où il a reconnu une double démocratisation de la gestion scolaire : l'élection au suffrage universel des membres du conseil des commissaires et l'installation d'une gestion participative interne.

Les multiples responsabilités qui incombent au système d'enseignement primaire et secondaire requièrent un personnel professionnel hautement qualifié. Selon Éthier (1989), la bureaucratie professionnelle caractérise les organisations de service. Les commissions scolaires emploient beaucoup de professionnels pour la réalisation de leur mission. La standardisation des compétences de ces professionnels assure la coordination du travail. Afin de respecter l'objectif que nous nous sommes fixé, nous allons nous en tenir aux responsabilités imposées à la direction générale.

\section{Les commissions scolaires d'aujourd'hui : mission et responsabilités}

La Loi sur l'instruction publique (L.I.P.) est fort explicite en ce qui a trait à la mission et aux obligations de la commission scolaire. Elle est une entreprise de services publics. Elle offre des services éducatifs aux personnes qui fréquentent ses établissements.

Par rapport aux entreprises privées, elle diffère, au moins, sur trois points. Il n'y a pas de client à l'école. Il y a des élèves qui viennent chercher des services éducatifs. Ceux-ci ne peuvent être dispensés sans que l'élève lui-même participe à l'acte éducatif. En effet, l'acte d'enseigner n'existe pas de façon isolée, il demande nécessairement son complément, l'acte d'apprendre. Dans l'établissement scolaire, l'enseignant et l'apprenant sont indissociablement liés. Impossible de faire autrement, sinon l'établissement scolaire n'existe pas. En deuxième lieu, les services offerts par l'établissement scolaire sont intangibles et ils doivent être personnalisés. Il n'y a pas deux personnes qui apprennent de la même manière. Ce n'est pas un service général, il doit être adapté à chaque personne. C'est ce qu'on désigne souvent sous le vocable enseignement individualisé. Ici, l'école se rapproche de l'hôpital. Enfin, un service public est une activité d'intérêt général. De ce fait, il doit être présent tout le temps et disponible à toutes les personnes. Enfin, il doit être de la même qualité en tout lieu.

Elle intervient dans un milieu déterminé et circonscrit par le gouvernement québécois où elle joue un rôle socio-économique très important. On soulignera, en effet, que les 72 commissions scolaires réparties en 17 régions administratives accueillaient en 2000-2001, 634708 élèves au préscolaire et au primaire, $363100 \mathrm{au}$ secondaire, 40584 à l'éducation des adultes et 58436 à la formation professionnelle. Pour la même année scolaire, elles dépensaient 5790751310 \$, si on ne tient pas compte des dépenses d'investissement, de financement, du transport scolaire, ainsi que certaines autres dépenses, afin d'offrir les services éducatifs à leurs clientèles.

De l'obligation générale d'offre de services éducatifs découlent des fonctions liées à la gestion des ressources humaines, matérielles et financières, ainsi qu'au transport scolaire. Enfin, elle a des obligations liées aux services à la communauté. 


\section{Directeur général et directeur général adjoint : profil, rôles et fonctions Rôles et fonctions}

Ce bref aperçu des obligations de la commission scolaire nous permet d'élaborer quelque peu sur le travail de son haut-fonctionnaire : le directeur général. La première obligation d'une commission scolaire consiste en l'engagement d'un directeur général et d'un directeur général adjoint ${ }^{4}$. Ces personnes ont pour fonctions d'assister le conseil des commissaires et le comité exécutif dans l'exercice de leurs fonctions et de leurs pouvoirs, d'assurer la gestion courante des activités et des ressources de la commission scolaire, de veiller à l'exécution des décisions du conseil des commissaires et du comité exécutif et d'exercer les autres tâches que leur confient le conseil et le comité exécutif. Les employés de la commission ainsi que les directeurs d'école ou de centre exercent leurs fonctions sous l'autorité du directeur général. Ceux qui sont affectés à une école ou à un centre sont sous l'autorité du directeur de l'école ou du centre.

En somme, il est évident que les devoirs et obligations du directeur général et de son adjoint sont ceux de la commission scolaire. Ainsi, l'Association des directeurs généraux des commissions scolaires affirme (2002) que «l'emploi de directeur général comporte la responsabilité totale de la gestion des activités des programmes et des ressources de l'organisme pour l'ensemble des unités administratives, des établissements et des champs d'activité ainsi que du suivi de l'exécution des décisions du conseil des commissaires et du comité exécutif, conformément aux dispositions législatives et réglementaires en vigueur".

Selon la même source, l'emploi de directeur adjoint peut comporter notamment les responsabilités suivantes:

- participer à l'élaboration des objectifs et des politiques de la commission scolaire;

- coordonner l'application des politiques de la commission en concertation avec les directions d'unités administratives dans des champs d'activités de nature administrative ou éducative;

- participe au comité consultatif des services aux élèves handicapés et aux élèves en difficulté d'adaptation ou d'apprentissage.

Ces personnes doivent posséder des qualités et des compétences associées aux rôles qu'elles sont appelées à exercer et que recouvre fort bien la nomenclature de Mintzberg (1984).

4. Il arrive que cette dernière fonction soit assumée par le secrétaire général de la commission scolaire. 


\section{Les rôles interpersonnels}

Le directeur général en autorité formelle, de par sa position statutaire, exerce les trois rôles interpersonnels :

\section{- Comme symbole}

À cause de la position qu'il détient au sein de son entreprise et de l'autorité formelle dont il est investi, le cadre est un symbole, ce qui lui impose des obligations. Certaines sont banales; d'autres demandent un certain doigté. Les obligations symboliques sont interpersonnelles; elles n'entraînent pas une activité appréciable de décision ou de traitement de l'information.

\section{- Comme leader}

Toute entreprise s'attend à ce que celui qui occupe la tête de son administration dirige, conseille et motive. À lui, revient la très grande responsabilité de créer l'atmosphère de l'entreprise. On s'attend à ce qu'il ait une vision énergique et qu'il la transmette à toute la hiérarchie.

\section{- Comme agent de liaison}

Quand on considère le rôle que joue le directeur général, on ne peut que constater qu'il entre très souvent en contact avec une foule de personnes ou de groupes oeuvrant à l'extérieur de son entreprise.

\section{Les rôles liés à l'information}

Grâce à ces rôles interpersonnels, le directeur général est dans une position privilégiée pour obtenir des informations aussi bien de l'extérieur, grâce à ses contacts avec des personnes situées hors de son organisation, que de l'intérieur, grâce à ses activités de chef de l'organisation. Il en résulte qu'il émerge comme le centre nerveux et le point-clef d'un certain nombre d'informations organisationnelles. De là proviennent les trois rôles liés à l'information.

\section{- Observateur actif}

Le directeur général est constamment à la recherche de l'information lui permettant de comprendre son entreprise et l'environnement dans lequel elle évolue. La complexité et l'incertitude de l'environnement exigent que le directeur général soit très attentif à ce qui s'y passe. Dans son étude, Mintzberg a dégagé l'information reçue par les directeurs généraux en cinq catégories: 1) les opérations internes, 2) les événements extérieurs, 3) les analyses, 4) les idées et les tendances, et 5) les pressions.

\section{- Diffuseur}

Étant donné le caractère direct et large de l'accès qu'il a à l'information, le directeur général doit la diffuser. Il joue ce rôle dans un double mouvement, en transmettant l'information dont il dispose à l'extérieur et à l'intérieur de son organisation, véhiculant ainsi des faits et des valeurs. 


\section{- Porte-parole}

Dans ce rôle, le directeur général transmet des informations à l'extérieur de son entreprise. C'est lui qui parle au nom de son organisation. Plusieurs groupes doivent être informés, notamment les membres du conseil des commissaires, les Autorités de tutelle de la commission scolaire et le public de manière plus large.

\section{Les rôles décisionnels}

La position remarquable du directeur général dans le circuit de l'information, son statut et son autorité le placent à un point central du système où sont élaborées les décisions stratégiques importantes. À cet égard, ses rôles décisionnels sont diversifiés :

\section{- Entrepreneur}

Tous les changements planifiés qui prennent place dans une organisation sont de la responsabilité du directeur général. Il en prend l'initiative et en assure la conception.

- Régulateur

Tout ne peut pas être prévu. Dans une organisation, il se produit inévitablement des situations inattendues. Le rôle du directeur général consiste à réguler ces situations afin d'éviter que l'inattendu vienne perturber la bonne marche de l'organisation.

\section{- Répartiteur de ressources}

L'élaboration d'une stratégie organisationnelle requiert des ressources. Sans ressources, aucune planification stratégique ne peut être élaborée. C'est notamment dans l'allocation et l'engagement de ressources, en fonction des acteurs et des situations, que la stratégie existe.

\section{- Négociateur}

La commission scolaire est une entreprise publique. Son directeur général est au centre des débats qui prennent place dans l'organisation, de manière formelle autant qu'informelle. Étant donné le rôle central qu'il joue dans l'organisation, c'est à lui que revient l'obligation de négocier avec d'autres organisations ou d'autres individus ou groupes d'individus.

Le modèle de Mintzberg nous aura permis de présenter une analyse systématique sinon détaillée du travail du directeur général de la commission scolaire (et de ses adjoints), dont la position entre l'autorité gouvernementale centrale et le lieu de réalisation des activités éducatives lui confère une très grande importance stratégique. 


\section{Profil des gestionnaires de la commission scolaire}

Sur la base de données statistiques du ministère de l'Éducation du Québec, en 2001-2002, sur les 61 présidences des conseils des commissaires francophones du Québec, 26 sont occupées par des femmes, soit un taux de féminisation de 42,62\%. Il faut noter que la région 16, la Montérégie, remporte la palme de féminisation avec un taux de $66,66 \%$.

Le poste de direction générale de ces commissions scolaires est occupé à 80,33\% par des hommes dans l'ensemble du Québec. La région 03, région de la Capitale, montre le plus haut taux de féminisation, à ce poste, soit $60,0 \%$.

Chez les commissaires élus, sur la base des données officielles, on n'observe pas de différence significative entre les hommes au nombre de 580 et les femmes au nombre de 561 qui occupent ce poste. Par contre, chez les commissaires désignés par les Comités de parents, on dénote un taux de féminisation de $64,4 \%$.

\section{Mécanismes et structures d'encadrement de l'exercice des activités des Directeurs Généraux et Directeurs Généraux Adjoints Textes législatifs et réglementaires}

Bien que la Loi sur l'instruction publique soit celle qui encadre les activités de la commission scolaire et, par le fait même, celles du DG et du DGA, bien d'autres définissent le travail de ces deux cadres. Ainsi, les politiques, les lois et les règlements relatifs aux relations du travail ont un impact très important sur le travail du DG et du DGA. D'ailleurs, les conditions de travail de ces deux cadres sont définies par décret gouvernemental; sans doute, peut-on penser qu'une certaine négociation a pris place entre le gouvernement et l'ADIGECS lors de l'établissement de ce décret. Il faut aussi savoir que toute la réglementation concernant la santé et la sécurité des personnes qui travaillent ou qui reçoivent des services éducatifs vient préciser leur travail. On peut aussi signaler toute la réglementation relative au transport scolaire. Dès lors, nous comprenons que la liste pourrait s'allonger, mais les exemples que nous venons de citer montrent jusqu'à quel point le travail du DG et du DGA est politiquement et légalement défini et encadré.

\section{Association des directeurs généraux des commissions scolaires (ADIGECS)}

Outre les lois et règlements déterminant de manière formelle les activités des directeurs généraux des commissions scolaires et les modes d'exercice de ces activités, il existe depuis maintenant trente ans une association, l'ADIGECS, regroupant les directeurs généraux, les directeurs généraux adjoints et les conseillers-cadres des commissions scolaires, soit au total 142 membres, selon les statistiques fournies par l'Association (2002). Pour des renseignements plus détaillés et par région administrative, le lecteur intéressé pourra consulter, en annexe, le tableau 4 relatif aux statistiques sur les membres de l'ADIGECS.

Nous référant aux textes constitutifs de l'ADIGECS, il ne fait aucun doute pour ses créateurs qu'elle est un organisme important à un double plan: social, parce qu'appelé à «influencer le développement des politiques d'éducation au Québec » et 
professionnel, parce que devant "promouvoir le statut et les intérêts professionnels de ses membres », en assurant d'une part, la qualité de l'exercice de la profession, en soutenant d'autre part, le développement et le perfectionnement de ses membres et en contribuant enfin à la détermination des normes professionnelles d'exercices de la fonction.

En vue de l'accomplissement de sa mission, l'ADIGECS, constituée en corporation par lettres patentes en vertu des dispositions de la Loi des compagnies, s'est dotée d'un règlement général créant diverses instances d'encadrement de l'exercice des activités de ses membres et définissant leurs attributions et compétences. Ainsi, pour ne mentionner que les plus importants, dans la première partie du Règlement, les articles 3.2, 3.7 et 4 définissent-ils respectivement les pouvoirs du Conseil d'administration, les pouvoirs du président et la composition, le rôle et les modes de fonctionnement du Conseil général de l'ADIGECS, tandis que l'article 5, de la partie II, définit l'appartenance des membres et leurs obligations, ce qui n'est pas sans lien avec son code d'éthique.

\section{Code d'éthique de l'ADIGECS}

L'ADIGECS a un code d'éthique qui lui est propre tout comme les commissions scolaires ont les leurs. Ils sont évidemment compatibles et en harmonie.

Comme il se doit, et sur la base des principes fondamentaux reliés au couple formé par la confiance du public et la crédibilité des membres, ce code d'éthique des l'ADIGECS se définit, selon une seule et même finalité mais dans une double perspective «servant de cadre de référence d'une part aux membres de l'Association dans l'exercice de leurs fonctions et responsabilités » et « servant de référence d'autre part aux membres dans leur action au sein de l'Association ".

Découlant et articulés autour de ces grands buts, les devoirs généraux des membres y sont définis (art. 3) ainsi que leurs lignes de conduite dans l'exercice de leurs fonctions (art. 4) et au sein de l'Association (art. 5).

De surcroît, un comité composé de trois membres désignés par le Conseil d'administration est mis en place en vue de promouvoir le code d'éthique professionnelle et de veiller au respect de son application. Ce comité dit d'éthique professionnelle peut, dans l'exercice de ses prérogatives, aller jusqu'à prendre des sanctions disciplinaires à l'encontre des membres fautifs.

Les éléments développés dans les sections qui précèdent sont des indices que les membres de l'ADIGECS jouissent d'une certaine autonomie dans l'exercice de leurs fonctions et responsabilités. Il n'en reste pas moins cependant, comme il a été déjà souligné, que cette autonomie est de manière générale balisée par des lois et des règlements et que l'action des directeurs généraux et de leurs adjoints est menée en étroite collaboration avec le Conseil des commissaires en ce qui concerne les fonctions de gestion et en partenariat avec le ministère de l'Éducation du Québec et d'autres organismes comme la Fédération des commissions scolaires du Québec en ce qui a trait notamment à l'élaboration de politiques pour le bon fonctionnement et le développement du secteur de l'éducation. Que peut-on dégager et conclure de ce qui précède globalement concernant le statut professionnel des directeurs généraux et de leurs adjoints? 


\section{Mise en perspective des faits relatifs aux DG et DGA par rapport aux modèles d'analyse de l'identité professionnelle}

Même en l'absence des données de l'enquête, les informations tirées des documents consultés et analysées précédemment nous permettent d'affirmer que les directeurs généraux des commissions scolaires répondent de manière claire à nombre de critères et de caractéristiques d'une profession ou d'un groupe professionnel, tels qu'ils ont été mis en relief dans le modèle d'analyse de l'identité professionnelle présenté plus haut.

La fonction de direction générale d'une commission scolaire a été créée par le législateur, dans le cours de l'évolution du système scolaire du Québec, pour répondre à des missions précises, relatives au fonctionnement et au développement du secteur de l'éducation aux niveaux préscolaire, primaire et secondaire ainsi que pour l'éducation des adultes et la formation professionnelle. Les actes professionnels, associés à cette fonction que sont appelés à exercer les directeurs généraux et les directeurs généraux adjoints sous la gouverne du Conseil des commissaires, s'articulent autour de la gestion de l'ensemble des ressources, notamment humaines, financières et matérielles, disponibles à la Commission scolaire en vue de la meilleure réalisation possible des buts des établissements scolaires et des centres de formation dont elle a la charge. Il y a lieu sans doute de spécifier qu'aux tâches de gestion proprement dite s'ajoute le volet extrêmement important des relations de la commission scolaire avec son environnement, les instances et institutions au sein du système éducatif d'une part et les instances et institutions des autres secteurs de la société d'autre part.

Il y a donc, exprimées de manière synthétique et formelle par les lois et les règlements de l'État mais aussi par d'autres vecteurs de la société civile, des représentations et des attentes claires vis-à-vis la fonction de direction générale d'une commission scolaire, fonction pour laquelle les aspirants et candidats doivent répondre à des exigences explicites en termes de compétences académiques et de comportements professionnels. Ces savoirs et savoir-faire, ces valeurs, attitudes et manières d'être ne s'acquièrent qu'au terme de processus assez longs en milieu de formation et de travail. Mais aussi, ils sont consolidés à travers des activités formelles et informelles qu'offre le groupe professionnel, l'ADIGECS, qu'ont créé voilà déjà trente ans les directeurs généraux des commissions scolaires, dans la foulée des événements qui ont marqué l'évolution du système scolaire québécois, notamment depuis le début des années 1960, avec la Réforme Parent.

Comme nous l'avons déjà dit plus haut, l'ADIGECS, sans être une corporation professionnelle au sens strict du Code des professions du Québec, répond sans aucun doute à l'ensemble des critères et attributs d'un regroupement professionnel. Elle souscrit à des standards et préalables de formation; elle a un statut définissant les fonctions et attributions de ses membres ainsi que les normes et règles de leur exercice; elle a enfin un code d'éthique qui prévoit des mécanismes de contrôle et de sanction de l'exercice de leurs fonctions par les membres. 
Il reste tout de même une exception notable, dans la mesure où il nous semblerait inapproprié de qualifier d'unique ou d'exclusifle service que rend le directeur général d'une commission scolaire à la société en accomplissant sa fonction. Tout en reconnaissant une certaine spécificité de l'éducation et de la gestion des organismes appelés à dispenser les services éducatifs, nous ne sommes pas d'avis que la gestion des ressources humaines, financières et matérielles est unique ou exclusive à l'éducation et essentiellement différente par rapport à cette fonction dans d'autres secteurs, dans les organismes de santé par exemple. Cela veut dire qu'à l'intérieur de la fonction générale de gestionnaire et des exigences de base en termes de pré-requis de formation et d'expérience, des substitutions sont tout à fait possibles entre divers secteurs, notamment si ces secteurs logent à la même enseigne de service public.

Par ailleurs, même quand le Règlement général de l'ADIGECS traite dans son article 5 de la Partie II de l'appartenance et des obligations des membres, en dehors d'une enquête auprès de ces derniers, nous ne sommes pas en mesure de statuer sur les dimensions personnelles associées à l'identité professionnelle des directeurs généraux des commissions scolaires, comme leur sentiment de compétence et d'estime de soi, leur sentiment d'identification et d'appartenance à leur groupe professionnel, leur appréciation propre de la pertinence théorique et pratique des exigences et attentes formulées à leur endroit.

\section{Conclusion}

Notre toute dernière observation nous amène à rappeler ici que l'ADIGECS à laquelle nous avons adressé une requête pour la passation de notre questionnaire auprès de ses membres n'a pas pu accéder à notre demande dans les délais qui nous auraient permis de disposer de données perceptuelles importantes pour un traitement large et approfondi du sujet. Nous avons en effet mis en relief tout au long de la deuxième section de notre texte, en ce qui concerne la constitution de l'identité personnelle et professionnelle, l'importance du soi, des représentations qu'il a de luimême et l'interprétation qu'il a des images et représentations que les autres se font de lui, identités personnelle et professionnelle étant étroitement associées. Et reprenant à notre compte cette formule très forte de Cohen-Scali (2000, p. 201), nous disons que "plus que les faits, les situations et les contextes professionnels, c'est l'interprétation qu'en font les individus qui participe à la structuration de l'identité professionnelle » et sur toutes ces choses, nous ne pouvons que spéculer, comme c'est le cas, au-delà des définitions théoriques des fonctions et responsabilités de DG et DGA tirées des documents, pour les pratiques et les contraintes d'exercice de leur fonction. Dans un tel contexte, cette conclusion ne peut être que partielle et provisoire.

Nous sommes en effet en mesure de conclure, sur la base des données présentées et analysées plus haut, que les directeurs généraux des commissions scolaires et leurs adjoints, s'ils ne forment pas encore un corps professionnel, sont en voie de le devenir. Ils se démarquent en tout cas fort bien par les fonctions qu'ils accomplissent dans un secteur d'activités à coup sûr spécifique, accompagnés sinon encadrés par 
un code d'éthique et par un organisme qui, à la longue, pourrait être reconnu comme une corporation professionnelle. Malgré tout, ce corps professionnel ne peut prétendre à l'unicité ou à l'exclusivité des services rendus par ses membres au même titre que les ordres professionnels, tels que définis par le Code des professions du Québec. Au-delà de certaines différences, liées à l'ampleur et à la diversité des tâches ainsi qu'au niveau des responsabilités, d'autres cadres oeuvrant au sein du secteur éducatif pourraient revendiquer le même statut professionnel. Il en est de même pour des cadres gestionnaires de niveau équivalent oeuvrant dans d'autres secteurs d'activités.

Il nous faut enfin souligner, même à l'étape où nous sommes, que la fonction de direction générale d'une commission scolaire et tout ce qui en découle, ayant vu le jour à la faveur d'une conjoncture dans le processus d'évolution du système scolaire, pourraient tout aussi bien disparaître dans un futur proche ou éloigné. Il n'en resterait pas moins cependant que la dispense des services éducatifs à la population, jeune et moins jeune, nécessiterait encore des cadres qualifiés pour la gestion des ressources requises en vue de la réalisation de cette mission, elle-même essentielle pour la société et base d'une profession qui, à coup sûr, existe déjà en germe et qui ne peut aller qu'en se consolidant.

\section{Références bibliographiques}

Anadon, Marta (2000). L'enseignement en voie de professionnalisation. Dans C. Gohier et al. L'enseignant: un professionnel, Québec, Presses de l'Université du Québec.

Association des directeurs généraux des commissions scolaires (2002). Les fonctions du DG et du DGA. htttp:/ /www.adigecs.qc.ca

Becker, H. (1962). The mature of a profession. Dans B.H. Nelson (dir.), Education for the professions. The Sixty-first Yearbook of the National Society for Study of Education, Chicago, University of Chicago Press.

Blau, Peter M. \& Richard Scott (1962). Formal Organisations: A Comparative Approach. San Francisco, Chandler Publishing Co.

Bourdieu, P. (1987). Espace social et pouvoir symbolique. Dans P. Bourdieu, Choses dites, Paris, Éditions de Minuit.

Carr-Saunderns, A.M. et P.A. Wilson (1933). The professions. Oxford, the Clarendon Press.

Cohen-Scali, V. (2000). Alternance et identité professionnelle. Paris, Presses Universitaires de France. 
Commission des écoles catholiques de Montréal. La gestion participative : rapport du Comité d'étude sur la gestion participative des écoles secondaires. Office des relations publiques de la C.E.C.M., 1976.

Conseil supérieur de l'éducation (1993). Rapport annuel sur l'état et les besoins de l'Éducation. La gestion de l'éducation: nécessité d'un autre modèle. Québec, les Publications du Québec.

Conseil supérieur de l'éducation (1991). Rapport annuel sur l'état et les besoins de l'Éducation. La profession enseignante: vers un renouvellement du contrat social. Québec, les Publications du Québec.

Desaulniers, M.-P. et al. (dir.) (1997). Les défis éthiques en éducation. Sainte-Foy (Québec), Presses de l'Université du Québec.

Dubar, Claude (1991 et 1996). La socialisation: construction des identités professionnelles et sociales. Paris, Armand Colin.

Durkheim, M.E. (1911). Éducation et sociologie. Paris, P.U.F., Le Sociologue, 1966.

Éthier, Gérard (1989). La gestion de l'excellence en éducation. Québec, Presses de l’Université du Québec.

Féger, R. (dir.) (1997). L'éducation face aux nouveaux défis. Actes du $4^{\mathrm{e}}$ Congrès des Sciences de l'éducation de langue française du Canada, Montréal, Éditions Nouvelles.

Flexner, A. (1915). Is social work a profession? dans School and Society, Chicago, juin.

Gohier, C. et al. (dir.) (1999 et 2000). L'enseignant, un professionnel. Québec, Presses de l'Université du Québec.

Gohier, C. et Christian Alin (dir.) (2000). Enseignant-formateur : la construction de l'identité professionnelle: recherche et formation. Paris, L'Harmattan.

Gohier, C., M. Anadon, J. Bouchard, B. Charbonneau et J. Chevrier (2001). La construction identitaire de l'enseignant sur le plan professionnel: un processus dynamique et interactif. Dans Revue des Sciences de l'éducation, Vol. XXVII, No 1, Montréal (Québec).

Goode, W. J. (1960). Encroachement, Charlatanism and the Emerging Profession: Psychology, Sociology and Medicine. dans American Sociological Review, Vol. 25, pp. 902-914.

Goode, W. J. (1957). Community within a Community: The Professions, American Sociological Review, Vol. 22, No 2, pp. 194-200.

Gouvernement du Québec, Ministère de l'Éducation (1979). L'école québécoise, énoncé de politique et plan d'action, Québec, Éditeur officiel du Québec.

Greenwood, E. (1977). Attributes of a Profession. dans R. Pavalko (dir.), Sociological Perspectives on Occupations, Ithaca, Peacoek. 
Gross, Edward (1958). Work and Society. New York, Thomas Cromwade.

Kirouac, Jacques (1976). Relations de deux structures administratives différentes sur le profil décisionnel dans les régionales scolaires du Québec. Thèse doctorale inédite, Université d'Ottawa.

Lessard, C. M. Perron et P. Bélanger (rédacteurs invités) (1993). La professionnalisation de l'enseignement et la formation des enseignants. Numéro thématique VR.XIX \#1, Revue des Sciences de l'éducation.

Lieberman, M. (1956). Education as a Profession. Englewoods, Cliffs (NJ), Prentice Hall Inc.

Mead, M. (1934). Mind, Self and Society: Form the Standpoints of a Social Behaviorist. Chicago, University of Chicago Press.

Mintzberg, Henry (1984). Le manager au quotidien: les dix rôles du cadre, Paris, Les éditions d'organisation.

Nelson, B.H. (dir.) (1962). Education for the Professions. The Sixty Yearbook of the National Society for Study of education, Chicago, University of Chicago Press.

Parsons, T. (1969). Professions, Polity and Society: Some General Considerations. dans T. Parsons (dir.), Politics and Social Structure, New York, The Free Press.

Québec (Province) (1968). Ministère de l'éducation. Direction générale de la planification. Les structures fonctionnelles des commissions scolaires régionales: définitions, principes, organisation administrative, structures et organigrammes. Document préliminaire.

Québec (Province) (1979). Ministère de l'éducation. L'école québécoise : énoncé de politique et plan d'action, Québec, Ministère de l'éducation.

Québec (Province) (1956). Rapport de la Commission royale d'enquête sur les problèmes constitutionnels, Québec Imprimeur de la Reine.

Québec (Province) (1963-1966). Rapport de la Commission royale sur l'enseignement dans la province de Québec. 5 volumes, 1 annexe et 1 index analytique, Imprimeur de la Reine, Québec Imprimeur de la Reine.

Tardif, M. et H. Ziarko (dir.) (1997). Continuité et ruptures dans la formation des maîtres au Québec. Québec, Presses de l’Université Laval.

Trottier, C. (1999). La création d'un ordre des enseignants et la professionnalisation de l'enseignement. Dans M. Tardif et C. Gauthier (dir.), Pour ou contre un ordre professionnel des enseignantes et des enseignants au Québec? Québec, Les Presses de l'Université Laval. 